\title{
GLOBAL MAPPING PROPERTIES OF RATIONAL FUNCTIONS
}

\author{
Cristina Ballantine ${ }^{1}$ and Dorin Ghisa ${ }^{2}$
}

\begin{abstract}
We investigate the fundamental domains of rational functions and provide visualizations for relevant examples. The fundamental domains give a thorough understanding of the global properties of the functions studied.
\end{abstract}

\section{Introduction}

Any rational function $f(z)$ can be viewed as the canonical projection of a branched covering Riemann surface $(\widehat{\mathbb{C}}, f)$ of the Riemann sphere $\widehat{\mathbb{C}}$. Indeed, $f$ is locally injective in the neighborhood of every point $z \in \widehat{\mathbb{C}}$, except for the points $z_{k}$, which are solutions of the equation $f^{\prime}(z)=0$ and the points $c_{j}$ which are multiple poles of $f$. In [Bar-G] we have studied global mapping properties of Blaschke products, showing that every Blaschke product $w=B(z)$ of degree $n$ induces partitions of $\widehat{\mathbb{C}}$ into $n$ sets whose interior is mapped conformally by $B$ onto $\widehat{\mathbb{C}} \backslash L$, where $L$ is a cut. Following [A, p. $98]$ we called these sets fundamental regions or domains.

The fundamental regions have played an important role in the theory of automorphic functions. In fact, a fundamental region of a group of transformations is a fundamental region of an automorphic function with respect to that group. These regions characterize the global mapping properties of automorphic functions. In this paper we show that any rational function $f$ has similar properties. Moreover, once the fundamental regions of $f$ are known, invariants of $f$ can be found, i.e. mappings $U_{k}$ of the Riemann sphere on itself such that, for every $z \in \widehat{\mathbb{C}}$, we have $f \circ U_{k}(z)=f(z)$. Obviously, the set of these invariants is a cyclic group of order $n$. They are the cover transformations (see [A-S, p. 37]) of $(\widehat{\mathbb{C}}, f)$ and we can extend the concept of automorphic function to such a group. Using this terminology, the main result of this paper shows that any rational function $f$ is an automorphic function with respect to the group of cover transformations of $(\widehat{\mathbb{C}}, f)$. The proof is constructive and we use the technique of simultaneous continuations developed in [Bar-G] in order to find fundamental regions for $f$.

To visualize the fundamental regions, we color a set of annuli centered at the origin of the $w$-plane in different colors with saturation increasing counter-clockwise (i.e., determined by the argument of each point) and brightness increasing outward (i.e., determined by the absolute value each point) and impose the same color, saturation and brightness to the pre-image of every point in these annuli.

\footnotetext{
${ }^{1}$ cballant@holycross.edu, College of the Holy Cross, USA

${ }^{2}$ dghisa@yorku.ca, York University, Canada
} 


\section{A Simple Example: Linear Fractional Transformations}

We visualize the linear fractional transformation

$$
w=f(z)=\frac{a z+b}{c z+d}, \quad \text { where } a d-b c \neq 0
$$

as follows.

Consider the circle $w=r e^{i \theta}(r$ is fixed $)$. Its pre-image by $f$ is $z(r, \theta)=\frac{-d w+b}{c w-a}$.

$$
\lim _{r \rightarrow 0} z(r, \theta)=-\frac{b}{a}, \text { and } \lim _{r \rightarrow \infty} z(r, \theta)=-\frac{d}{c} .
$$

If $r$ is small, the pre-image is a circle containing $-\frac{b}{a}$. If $r$ is large, the pre-image is a circle containing $-\frac{d}{c}$. If $r=\left|\frac{a}{c}\right|$, the pre-image of the circle is a line (perpendicular to the line through $-\frac{b}{a}$ and $-\frac{d}{c}$, since $f$ is conformal).

Example 1: $f(z)=\frac{(2+3 i) z+(1-i)}{(1+2 i) z+(-1+4 i)}$.

The pre-images of the annuli
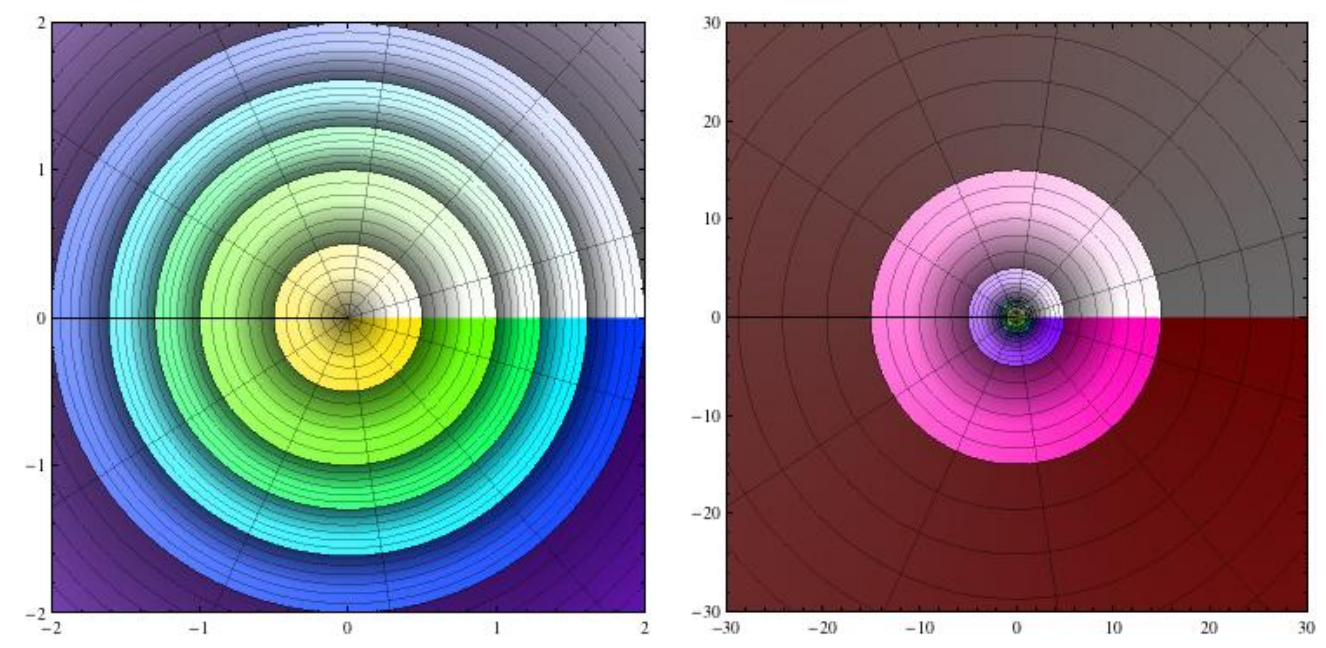

under the mapping $f$ are shown below. 

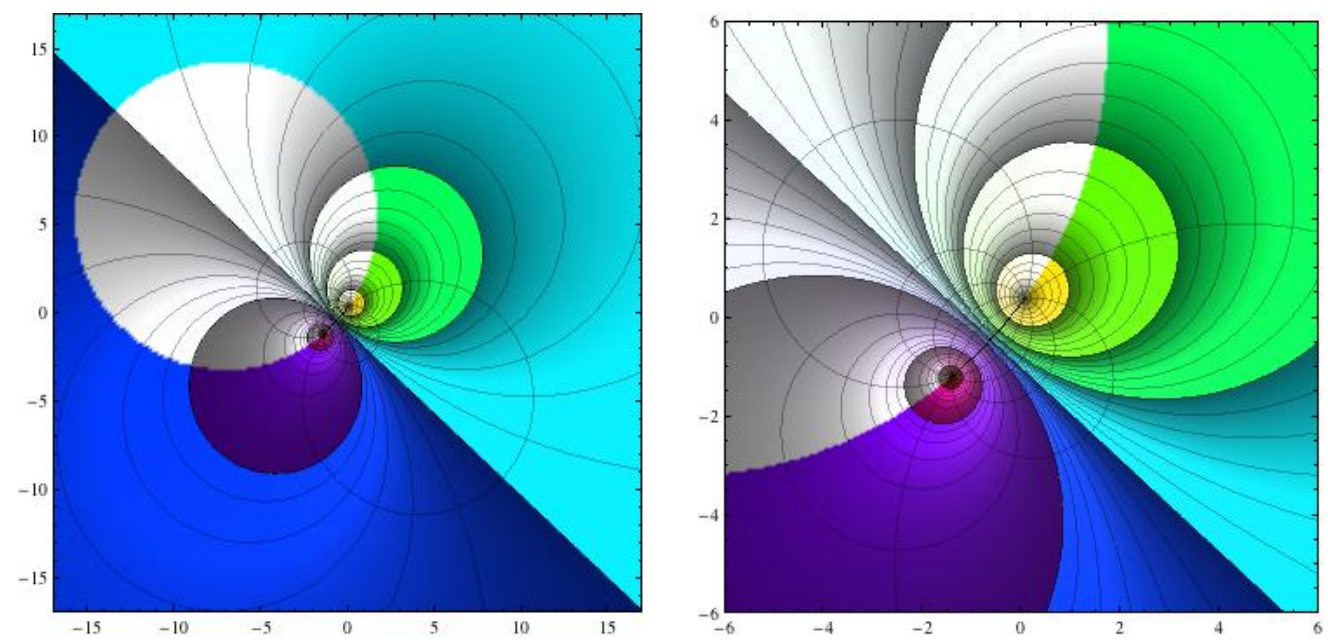

We notice that $f$ has a single fundamental domain.

For reasons of clarity, in the images above we provide a zoom of the colored annuli and of the fundamental domains.

\section{Mapping Properties of the Second Degree Rational Func- tions}

A study of the second degree rational functions can be found in [N, p.266]. We use Nehari's results in order to illustrate some of the mapping properties of these functions. The main result found in $[\mathrm{N}]$ relevant to this topic is that any mapping $w=f(z)=\frac{a_{1} z^{2}+a_{2} z+a_{3}}{b_{1} z^{2}+b_{2} z+b_{3}}$ can be written under the form

$$
f(z)=S_{2} \circ T \circ S_{1}
$$

where $S_{1}$ and $S_{2}$ are Möbius transformations and $\eta=T(\zeta)=\zeta^{2}$. Indeed, to prove this statement we only need to determine six essential parameters of the two unknown Möbius transformations $S_{1}$ and $S_{2}$ such that (1) is true, which is allays possible.

The function $\zeta=S_{1}(z)$ transforms the $z$-plane into the $\zeta$-plane, such that a circle (see Example 3 below) or a line $L$ (see Example 2 below) corresponds to the real axis from the $\zeta$-plane. The function $\eta=T(\zeta)=\zeta^{2}$ transforms each one of the upper and the lower half-planes of the $\zeta$-plane into the whole $\eta$-plane with a cut alongside the real half-axis. Finally, the function $w=S_{2}(\eta)$ transforms the $\eta$-plane into the $w$-plane and the real half-axis into an arc of a circle or a half line $L^{\prime}$. Summing up, $f$ maps conformally each one of the two domains determined by $L$ onto the whole $w$-plane with a cut alongside $L^{\prime}$. Thus, for such a function $f$, the fundamental domains can allays be taken the two domains mapped by $S_{1}$ onto the upper and the lower half planes.

Example 2: We illustrate the case where $f(z)=\frac{(1+i) z^{2}+4 z+1-i}{(2+i) z^{2}+6 z+2-i}$. In this case $S_{1}(z)=$ 
$\frac{z+1}{z-i}$ and $S_{2}(z)=\frac{z+i}{2 z+i}$. The pre-image of the real axis under $S_{1}$ is the line $z=\frac{1+t i}{t-1}$ shown below.

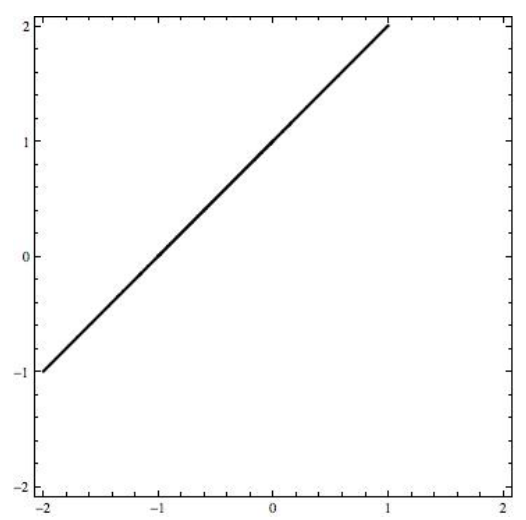

The image of the positive real half axis under $S_{2}$ is the semicircle of radius 0.25 centered at 0.75 .

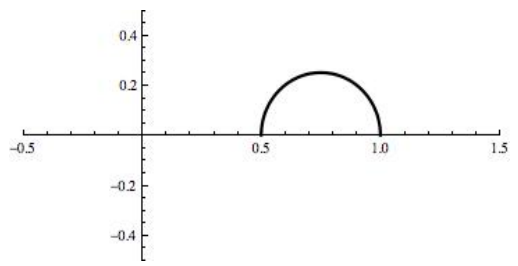

For the visualization, we consider colored annuli centered at $(0.75,0)$.

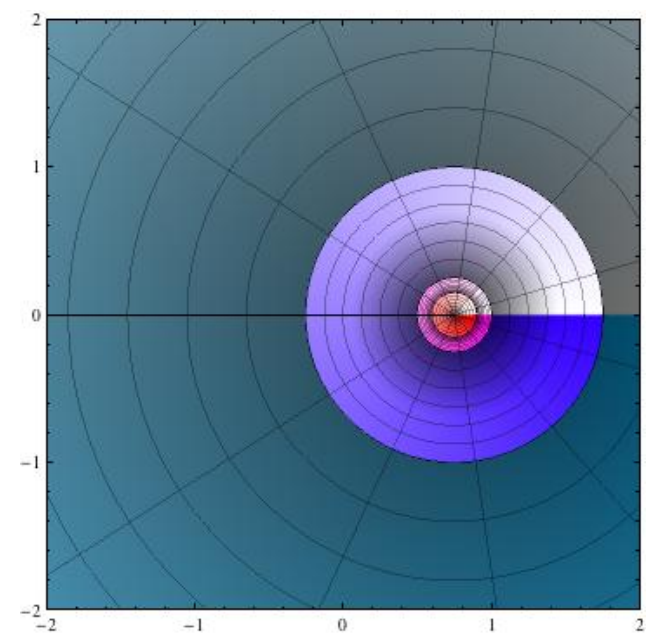


We visualize the fundamental domains of $f$ in Figure 1(a), by considering pre-images of these annuli under $f$.

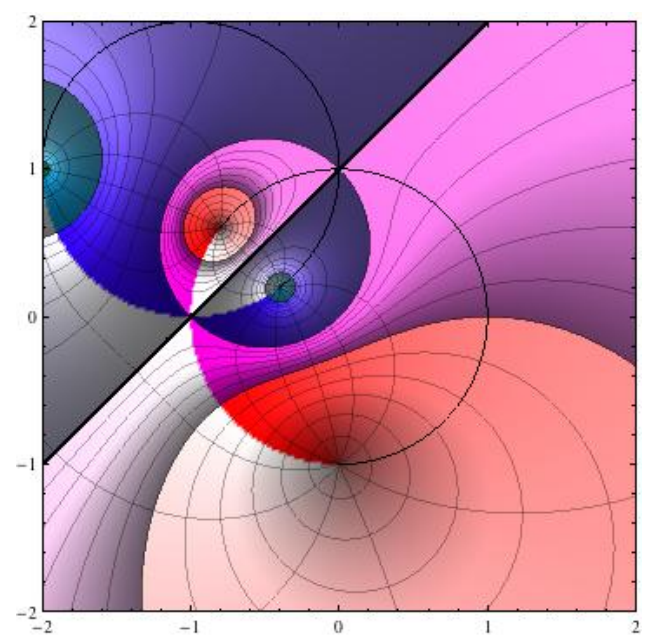

Note: $f$ has two funadamental domains. They are precisely the regions delimited by the preimage $L$ of the real axis under $S_{1}$.

Example 3: We illustrate the case $f(z)=\frac{(8-17 i)+(6-16 i) z-(9-9 i) z^{2}}{(8-19 i)+(16-14 i) z-(14-21 i) z^{2}}$.

Then, $S_{2}$ is the same Möbius transformation as in the previous example and $S_{1}$ is the Möbius transformation illustrated in the previous section.

Thus, $S_{1}(z)=\frac{(2+3 i) z+(1-i)}{(1+2 i) z+(-1+4 i)}$ and $S_{2}(z)=\frac{z+i}{2 z+i}$.

The pre-image of the real axis under $S_{1}$ is the circle shown below.

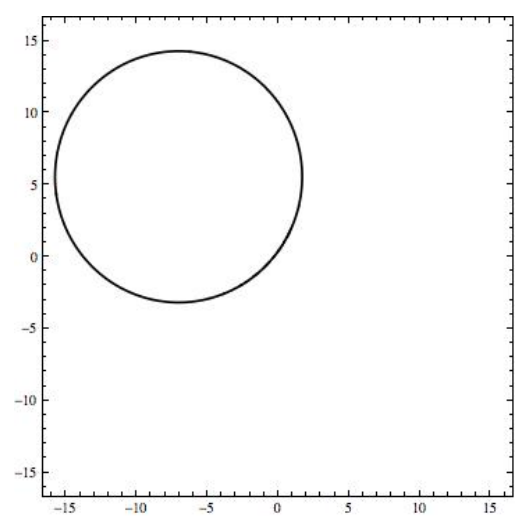

The pre-images of the annuli centered at 0.75 


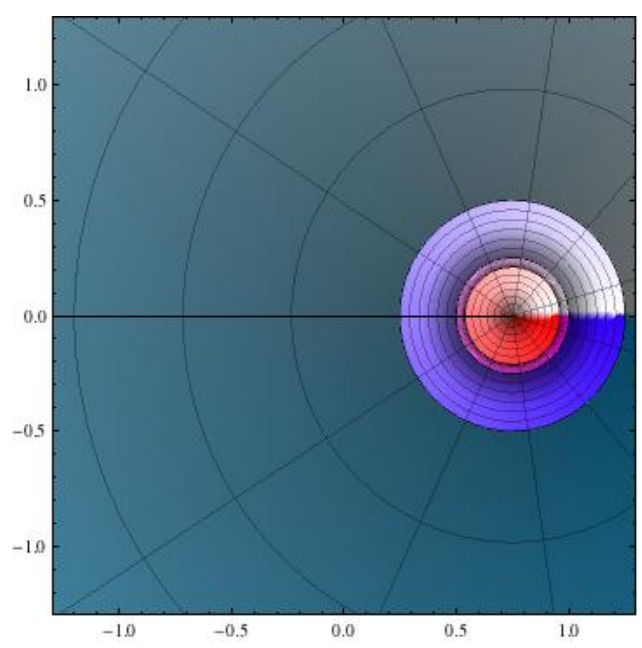

under the mapping $f$ are:
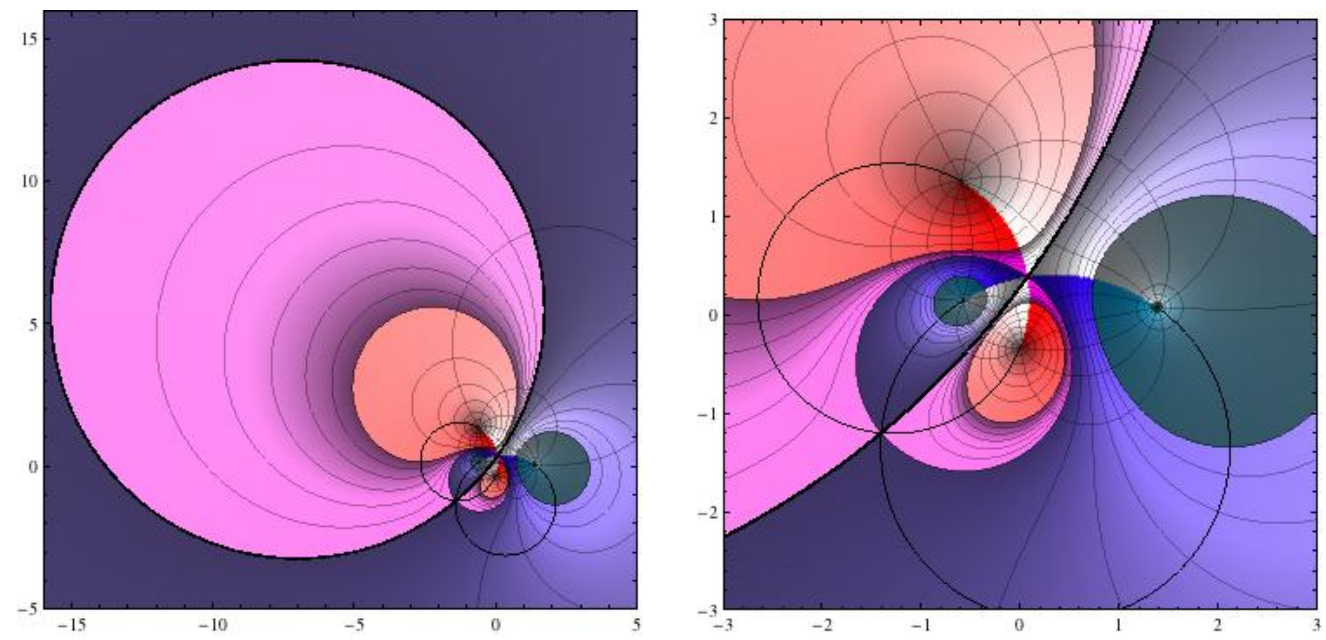

Note: $f$ has two fundamental domains: the interior of the disk whose boundary is the pre-image of the real axis under $S_{1}$ and the exterior of this disk.

\section{Mapping properties of Blaschke Quotients}

In [B-G] we studied the mapping properties of Blaschke quotients $B$ of a special type, namely such that for every $z \in \widehat{\mathbb{C}}, B \circ h(z)=h \circ B(z)$, where $h(z)=-1 / \bar{z}$. Such a rational function has the particularity that its poles and zeros appear in pairs which are opposite to each other and if $z_{k}$ is a pole of order $p$ of $B$, then $1 / \bar{z}_{k}$ is a zero of order $p$ of $B$ and vice-versa. The point $z=0$ is a zero or a pole of $B$ of an odd order and therefore $\infty$ is a pole, respectively a zero, of the same order.

The main result of $[\mathrm{B}-\mathrm{G}]$ shows that, for a Blaschke quotient of degree $n$ of such a type, there is a partition of $\widehat{\mathbb{C}}$ in $2 n$ simply connected sets such that the interior of each one of them is mapped 
conformally by $B$ either on the open unit disc (i-set), or on the exterior of the closed unit disc (e-set). The interior of the union of an i-set and an adjacent e-set is mapped conformally by $B$ on the Riemann sphere with a slit. The map is continuous (with respect to the spheric metric) on the borders, except for the branch points. Here, we prove that a similar property holds for any finite Blaschke quotient.

Let $B(z)=B_{1}(z) / B_{2}(z)$ be a Blaschke quotient of degree $n$, i.e. the quotient of two finite Blaschke products $B_{1}$ and $B_{2}$ of degrees $n_{1}$, respectively $n_{2}$, such that $\max \left\{n_{1}, n_{2}\right\}=n$. The function $B$ is locally injective, except for the set of points $H_{1}=\left\{b_{1}, b_{2}, \ldots, b_{m}\right\}$, which are solutions of the equation $B^{\prime}(z)=0$. Consequently, $(\widehat{\mathbb{C}}, B)$ is a branched covering Riemann surface of $\widehat{\mathbb{C}}$ having $H_{1}$ as set of branch points. In other words, $\left(\widehat{\mathbb{C}} \backslash H_{1}, B\right)$ is a smooth covering Riemann surface of $\widehat{\mathbb{C}}$.

Theorem 1 For every Blaschke quotient $B$ of degree $n$ there is a partition of $\widehat{C}$ into $n$ sets symmetric with respect to the unit circle whose interior $\Omega_{k}$ is mapped each one conformally by $B$ on $\widehat{\mathbb{C}} \backslash L$, where $L$ is a cut. Moreover, $B: \bar{\Omega}_{k} \rightarrow \widehat{C}$ is surjective.

Proof: Let $H_{2}=\left\{z_{1}, z_{2}, \ldots, z_{n}\right\}$ be the solutions of the equation $B(z)=e^{i \theta}$, where $\theta \in R$ has been chosen such that $H_{1} \cap H_{2}=\emptyset$. It is obvious that such a choice is always possible. Since the image of the unit circle by $B$ is the unit circle, at least one of the points $z_{k}$ belongs to the unit circle. Also, since $B(1 / \bar{z})=1 / \overline{B(z)}$, the solutions which are not on the unit circle, must be two by two symmetric with respect to the unit circle.

If we perform simultaneous continuation from every $z_{j}$ over the unit circle (starting from $e^{i \theta}$ ), we obtain $\operatorname{arcs} \gamma_{j, j^{\prime}}$ starting at $z_{j} \in H_{2}$ and ending at some point $z_{j^{\prime}} \in H_{2}$. Some of these arcs might cross each other, but this can happen only at the points in $H_{1}$ since these are the only points where the injectivity of $B(z)$ is violated.

Let $W=\left\{w_{1}, w_{2}, \ldots, w_{p}\right\}$, where $w_{k}=B\left(b_{k}\right),\left|b_{k}\right|<1, b_{k} \in H_{1}$ and $w_{k}$ are not points of intersection of $\gamma_{j, j^{\prime}}$. We connect $e^{i \theta}, w_{1}, \ldots, w_{p}$ by a polygonal line $\Gamma$ with no self intersection and perform simultaneous continuation over $\Gamma$ from all $z_{j} \in H_{2}$. The domains bounded by the pre-image of $\Gamma$ and the arcs $\gamma_{j, j^{\prime}}$ are mapped by $B$ either on the unit disc (i-domains) or on the exterior of the unit circle (e-domains). Indeed, every one of these domains $\Omega_{j, j^{\prime}}$ is bounded by an arc $\gamma_{j, j^{\prime}}$ whose image by $B$ is the unit circle, and by an arc having the end points in $z_{j}$ and $z_{j^{\prime}}$ whose image by $B$ is a part of $\Gamma$. The previous affirmation follows from the conformal correspondence theorem (see $[\mathrm{N}$, p. 154]). It is obvious that every i-domain has a symmetric e-domain with respect to the unit circle and vice-versa. An i-domain and an adjacent e-domain are always separated by an arc $\gamma_{j, j^{\prime}}$ and their union to which the open $\gamma_{j, j^{\prime}}$ is added as a point set constitutes a fundamental domain $\Omega_{j}$ of $B$. If we denote $L=\Gamma \cup \widetilde{\Gamma}$, where $\widetilde{\Gamma}$ is the symmetric of $\Gamma$ with respect to the unit circle, then it is obvious that $B$ maps conformally every $\Omega_{k}$ on $\widehat{\mathbb{C}} \backslash L$ and the mapping $B: \bar{\Omega}_{k} \rightarrow \widehat{\mathbb{C}}$ is surjective, which completely proves the theorem.

$$
\begin{aligned}
& \text { Example 4: Let } a_{1}=\frac{1}{4} e^{\frac{\pi i}{6}}, a_{2}=\frac{1}{3} e^{-\frac{\pi i}{5}} \text { and } b=\frac{1}{2} e^{\frac{2 \pi i}{3}} . \\
& B_{1}(z)=\left(\frac{\overline{a_{1}}}{\left|a_{1}\right|} \frac{z-a_{1}}{\overline{a_{1}} z-1}\right)^{2} \cdot \frac{\overline{a_{2}}}{\left|a_{2}\right|} \frac{z-a_{2}}{\overline{a_{2}} z-1}
\end{aligned}
$$




$$
B_{2}(z)=\left(\frac{\bar{b}}{|b|} \frac{z-b}{\bar{b} z-1}\right)^{2} .
$$

Then,

$$
B(z)=\frac{B_{1}(z)}{B_{2}(z)}=\frac{e^{-\frac{4 i \pi}{5}}\left(-\frac{1}{4} e^{\frac{i \pi}{6}}+z\right)^{2}\left(-\frac{1}{3} e^{-\frac{i \pi}{5}}+z\right)\left(-1+\frac{1}{2} e^{-\frac{2 i \pi}{3}} z\right)^{2}}{\left.\left(-\frac{1}{2} e^{\frac{2 i \pi}{3}}+z\right)^{2}\left(-1+\frac{1}{4} e^{-\frac{i \pi}{6}} z\right)\right)^{2}\left(-1+\frac{1}{3} e^{\frac{i \pi}{5}} z\right)}
$$

is a Blaschke quotient of degree 5 .

$H_{1}=\{0.216506+0.125 i,-1 .+1.73205 i, 0.162638+0.986686 i, 0.254261-0.0769968 i,-0.994981-$ $0.100059 i, 3.6026-1.09096 i\}$.

A polygonal line $L$ passing though the images of the branch points is shown below.
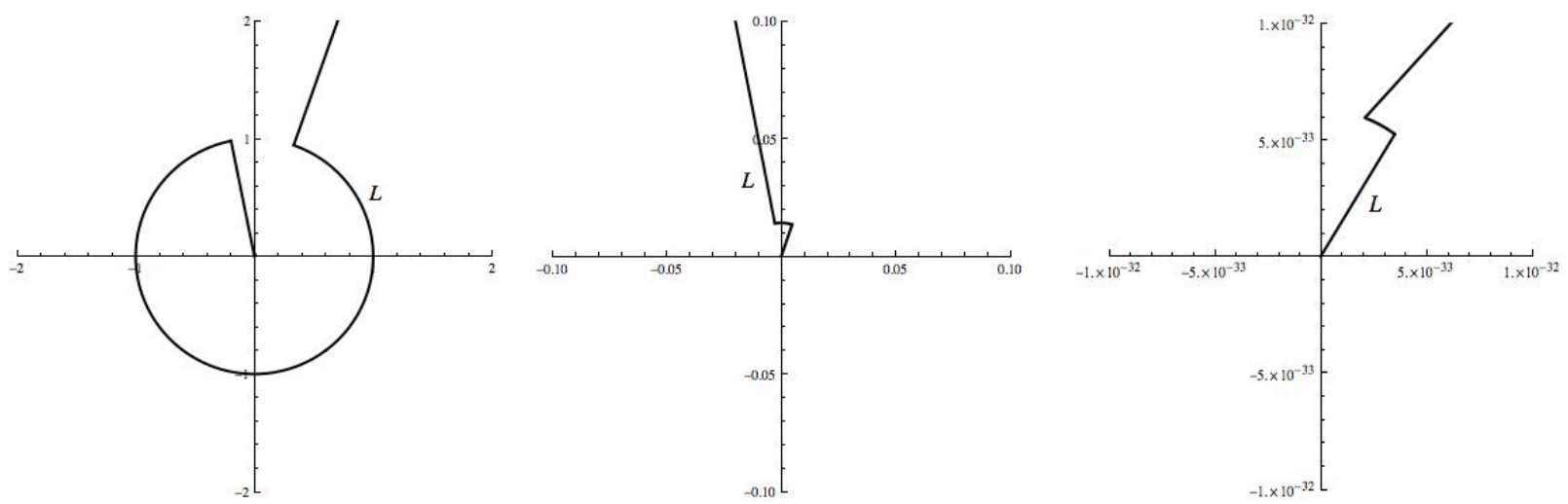

The pre-image of $L$ under $B$ is shown below.
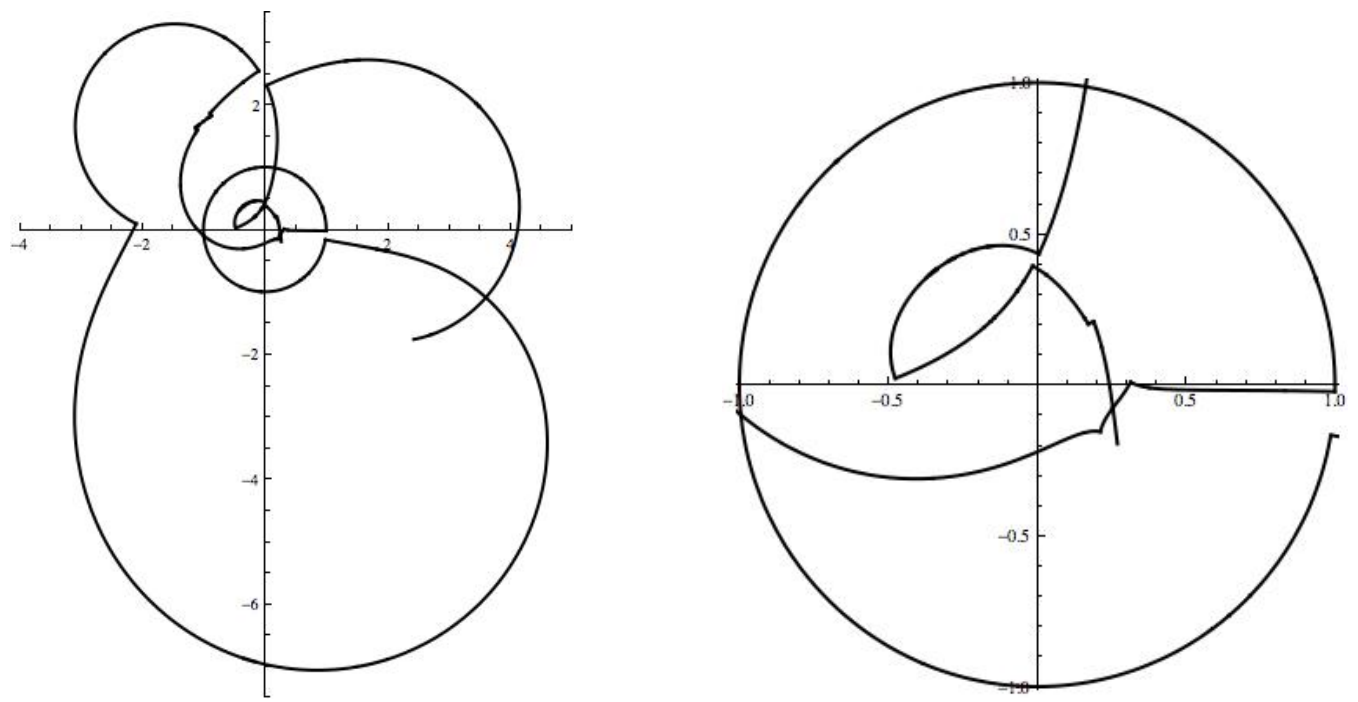
We consider a collection of colored annuli.
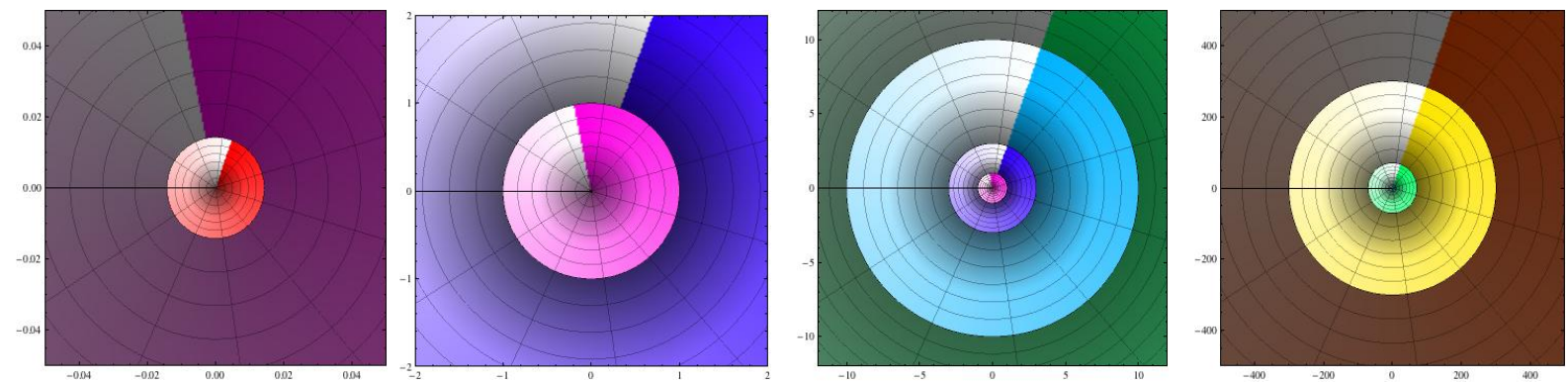

The pre-image of these annuli under $B$ are shown below.
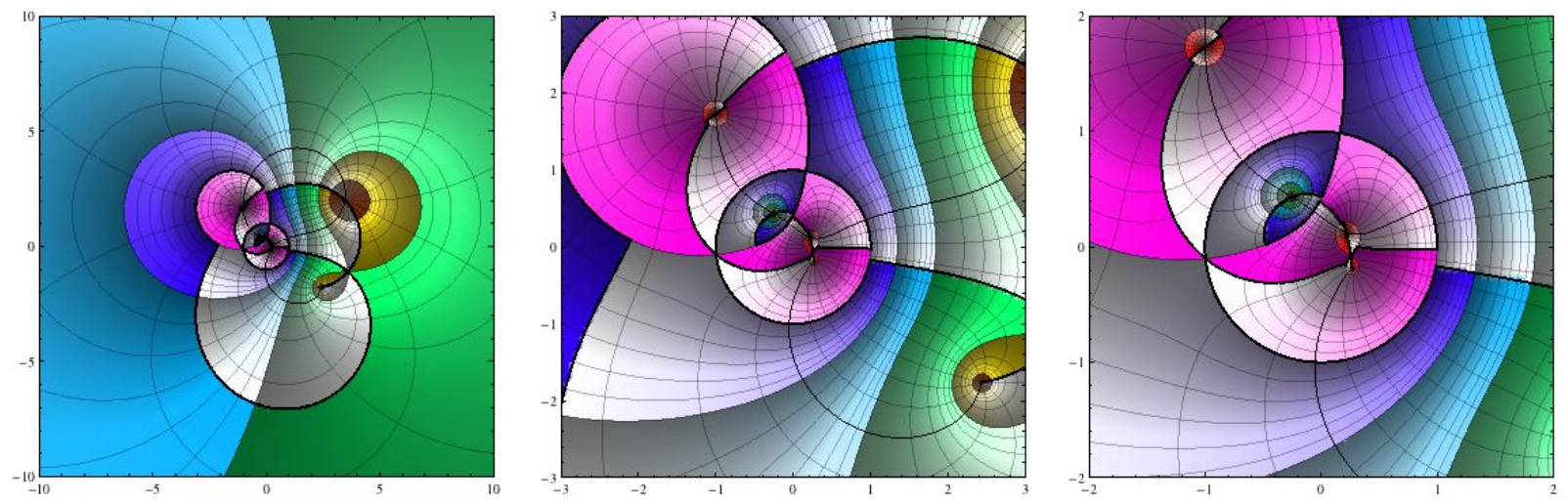

For a better view, each figure above shows several zoomed images.

In the next section we show that a similar property is true for any rational function.

\section{$5 \quad$ Mapping Properties of Arbitrary Rational Functions}

Let $w=f(z)$ be a rational function with zeros $a_{1}, a_{2}, \ldots, a_{p}$ and poles $b_{1}, b_{2}, \ldots, b_{q}$. Let $\alpha_{i}$ be the multiplicity of $a_{i}$ and $\beta_{j}$ be the multiplicity of $b_{j}$. Then, the degree of $f$ is $n=\max \{u, v\}$, where $u=\alpha_{1}+\alpha_{2}+\ldots+\alpha_{p}$ and $v=\beta_{1}+\beta_{2}+\ldots+\beta_{q}$.

If $\lim _{z \rightarrow \infty} f(z)=0, n=v$ and $a_{0}=\infty$ is said to be a zero of multiplicity $\alpha_{0}=n-u$ of $f$. If $\lim _{z \rightarrow \infty} f(z)=\infty, n=u$ and $b_{0}=\infty$ is said to be a pole of multiplicity $\beta_{0}=n-v$ of $f$.

Theorem 2 Every rational function $f$ of degree $n$ defines a partition of $\widehat{\mathbb{C}}$ into $n$ sets whose interior is mapped conformally by $f$ on $\widehat{\mathbb{C}} \backslash L$, where $L$ is a cut. The mapping can be analytically extended to the boundaries, except for a number $\leq n$ of common points $z_{j}$ of those boundaries in the neighborhood of which $f$ is of the form

(i) $f(z)=w_{j}+\left(z-z_{j}\right)^{k} h(z)$, when $f\left(z_{j}\right)=w_{j}$,

(ii) $f(z)=\left(z-z_{j}\right)^{-k} h(z)$, when $f\left(z_{j}\right)=\infty$, 
(iii) $f(z)=z^{-k} h(z)$, when $z_{j}=\infty$ and $f(\infty)=\infty$, with $h(z)$ analytic and $h\left(z_{j}\right) \neq 0, k \geq 2$. In other words, ( $\left.\widehat{\mathbb{C}}, f\right)$ is a branched covering Riemann surface of $\widehat{\mathbb{C}}$ and the branch points are $z_{j}$.

Proof: Since $\lim _{z \rightarrow a_{j}} f(z)=0$, we can find a positive number $r$ small enough such that the pre-image $\Gamma$ of the circle $\gamma_{r}$ centered at the origin and of radius $r$ will have disjoint components $\Gamma_{j}$, each containing just one zero $a_{j}$. If $\infty$ is a zero of $f$, then the respective component $\Gamma_{0}$ must be traversed clockwise, in order for $\infty$ to remain on its left. We understand by the domain bounded by $\Gamma_{0}$ (if $\Gamma_{0}$ exists) that component of $\widehat{\mathbb{C}}$ defined by $\Gamma_{0}$ which contains $\infty$. For the opposite orientation of $\Gamma_{0}$ we have a curve containing all the other components $\Gamma_{j}$.

Moreover, we can choose the above $r$ such that $f^{\prime}(z)=0$ has no solution in the closed domain bounded by $\Gamma_{j}$ except maybe for $a_{j}$. Then, for an arbitrary $\theta \in R$, the equation $f(z)=r e^{i \theta}$ has exactly $\alpha_{j}$ distinct solutions on $\Gamma_{j}$. Now, consider the pre-image by $f$ of the ray inside $\gamma_{r}$ determined by $r e^{i \theta}$. In the domain bounded by $\Gamma_{j}$ it consists of a union of $\alpha_{j}$ Jordan arcs having in common only the point $a_{j}$ and connecting $a_{j}$ to the solutions of $f(z)=r e^{i \theta}$ on $\Gamma_{j}, j=0,1,2, \ldots, p$ (see [A, p. $131-133])$.

Let $c_{k}, k=1,2, \ldots, m$, be the solutions of the equation $f^{\prime}(z)=0$ external to all $\Gamma_{j}$, and let $w_{k}=f\left(c_{k}\right)=r_{k} e^{i \theta_{k}}$. Suppose that $r_{1} \leq r_{2} \leq \ldots \leq r_{m}$. When $r_{k}=r_{k+1}$, then we take $\theta_{k}<\theta_{k+1}$, for every $k$. We perform simultaneous continuation starting from all $a_{j}$ over a curve $L$ from the $w$-plane in the following way. We take first the pre-image by $f$ of the segment from 0 to $r_{1} e^{i \theta_{1}}$. This is a union of arcs, $\alpha_{j}$ of which are starting in $a_{j}, j=0,1,2, \ldots, p$. At least one of these arcs is connecting one of the $a_{j}$ with $c_{1}$. If $r_{1}=r_{2}$, then we take the pre-image of the shortest arc between $w_{1}$ and $w_{2}$ of the circle centered at the origin and having the radius $r_{1}$ (if $w_{1}=-w_{2}$, we go counter-clockwise on that circle), etc. If $r_{k}<r_{k+1}$, we take the pre-image by $f$ of the union of the arc of circle centered at the origin and having the radius $r_{k}$, between $w_{k}$ and $r_{k} e^{i \theta_{k+1}}$, and the segment between this last point and $w_{k+1}$. After the point $w_{m}$ has been reached, if $f$ has at least one multiple pole, we take the pre-image of the ray from $w_{m}$ to $\infty$. If $f$ has no multiple pole, then the end of $L$ is $w_{m}$ and therefore $L$ is a finite path. In this way we build in a few steps the path $L$ and the simultaneous continuation over $L$ starting from all $a_{j}$. The continuation arcs can have in common only points $a_{k}, b_{k}$ or $c_{k}$, and all $b_{k}$ and $c_{k}$ are reached by several pre-image arcs. Indeed, if two such arcs meet in a point $c$, then they are both mapped by $f$ on the same sub-arc of $L$ starting in $f(c)$. One of the following four situations may happen:

a) $f(c)=0$ and $f^{\prime}(c)=0$, hence $c$ coincides with a multiple zero $a_{k}$. Then $f$ has the expression (i) with $w_{0}=0$ in a neighborhood of $c=z_{j}$.

b) $f(c) \neq 0$ and $f^{\prime}(c)=0$, hence $c$ coincides with a $c_{k}$. Then $f$ has the expression $(i)$ with $w_{0}=f(c)$ in a neighborhood of $c=z_{j}$.

c) $f(c)=\infty$ and $c$ is a multiple pole $b_{k}$ of $f$. Then $f$ has the expression $(i i)$ in a neighborhood of $c=b_{k}=z_{j}$.

d) $c=\infty$. Then $f$ has the expression (iii) in a neighborhood of $\infty$.

On the other hand, every $c_{k}$ and $b_{k}$ must be reached by some continuation arcs, since $f\left(c_{k}\right) \in L$ and $f\left(b_{k}\right) \in L$. More exactly, there are as many continuation arcs starting in $c_{k}$ as the multiplicity of $c_{k}$ as zero of the equation $f^{\prime}(z)=0$ and there are as many continuation arcs starting in $b_{k}$ as the multiplicity of $b_{k}$ as a pole of $f$. The arcs starting in simple zeros of $f$ border exactly $n$ 
bounded and/or unbounded domains $\Omega_{k}$ (fundamental domains) which are mapped conformally by $f$ on the $w$-plane from which the curve $L$ has been removed. This is a corollary of the boundary correspondence theorem (see [N, p. 154]). If we denote by $\bar{\Omega}_{k}$ the closure of $\Omega_{k}$, then it is obvious that $\widehat{\mathbb{C}}=\cup_{k=1}^{n} \bar{\Omega}_{k}$. With the notation $A_{k}=\bar{\Omega}_{k} \backslash \cup_{j=1}^{k-1} \bar{\Omega}_{j}$ we have the partition in the statement of the theorem.

Example 5: $f(z)=\frac{z^{3}(z+2)}{(z-i)^{4}(z+3-i)^{3}}$

We consider the polygonal line $L$ passing through each image of the zeroes of $f^{\prime}$

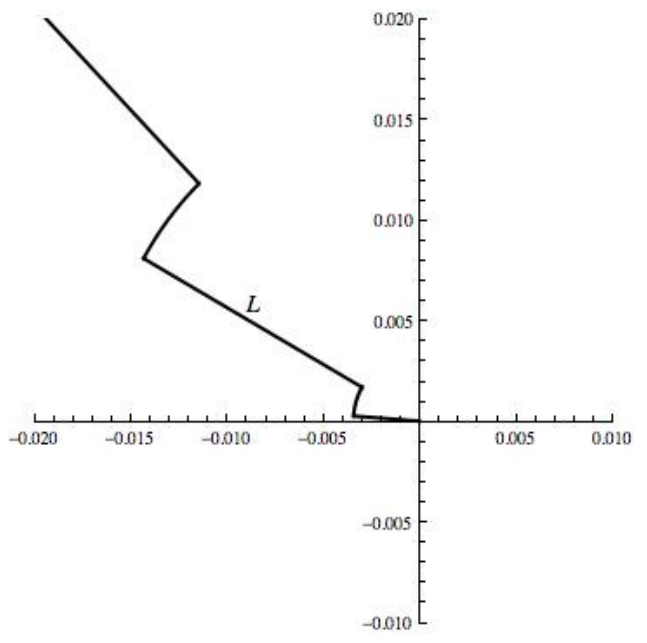

whose pre-image under $f$ is:
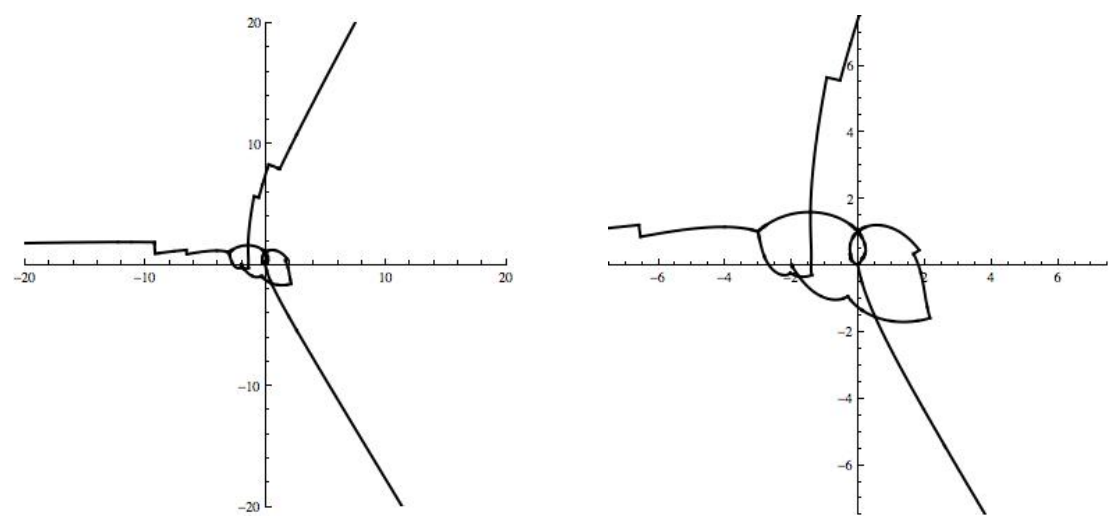

Below we consider a collection of colored annuli. In this case the saturation of the annuli increases starting at the polygonal line $L$. 

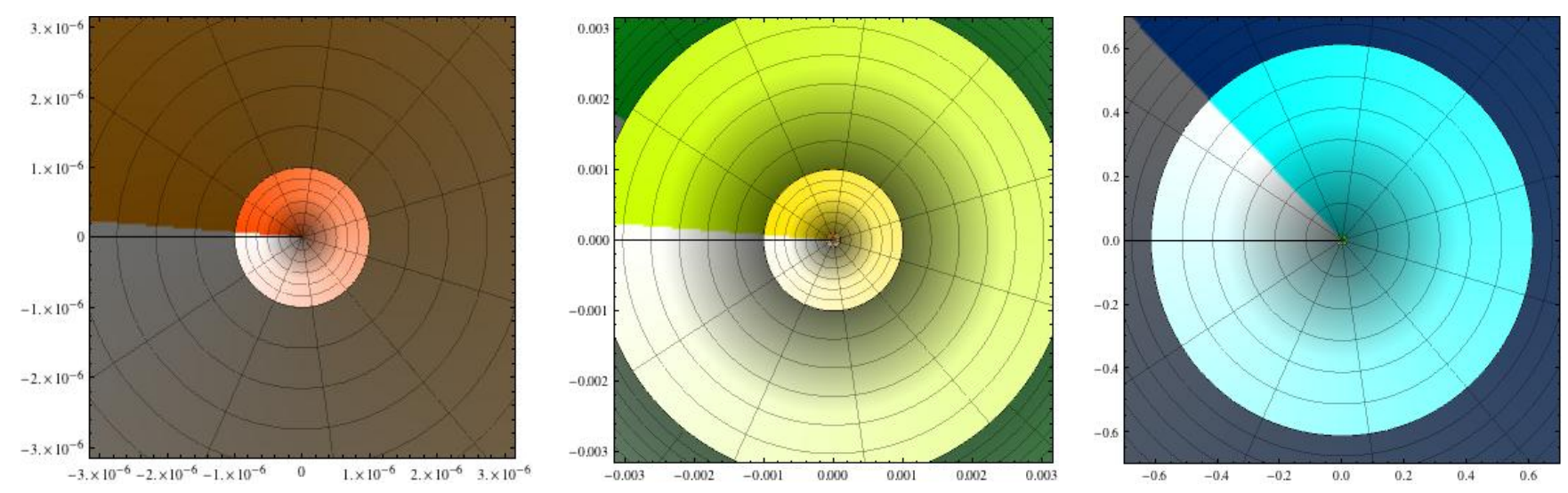

Their image under $f$ is shown below.
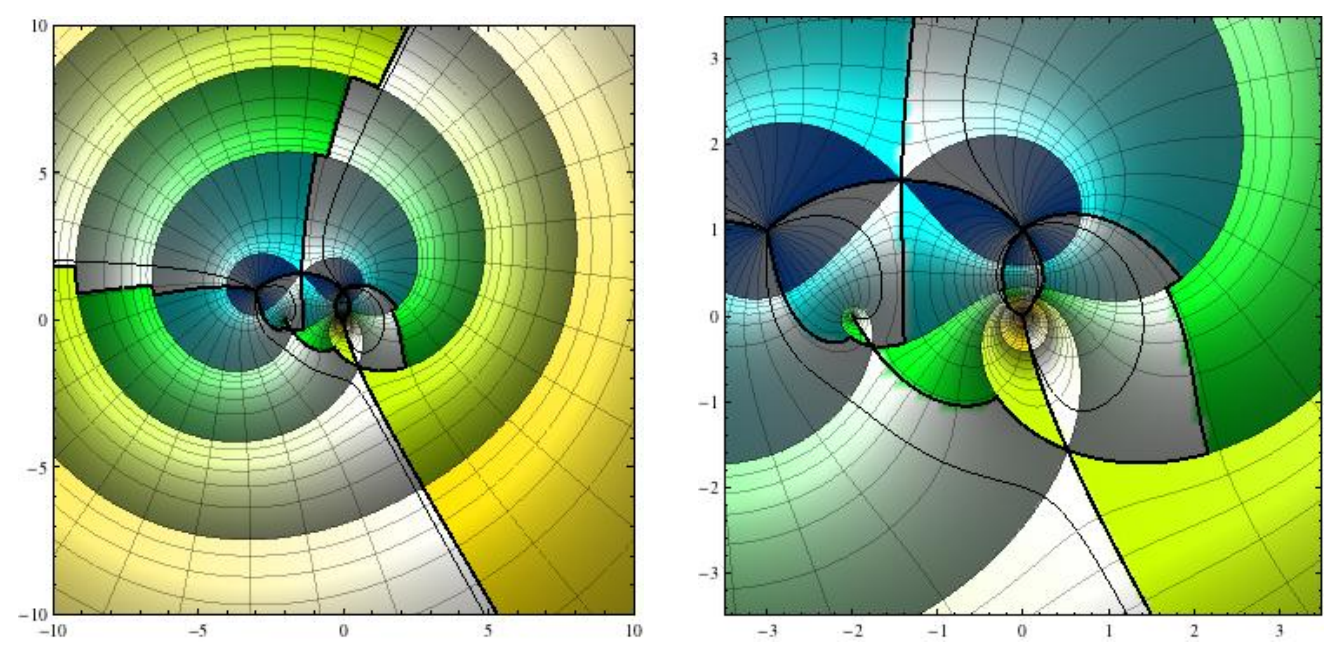

Finally, we examine the case in which $f$ is a polynomial of degree $n$. Then the unique pole of $f$ is $\infty$ and it has multiplicity $n$. Hence, the ray from $w_{m}$ to $\infty$ has as pre-image $n$ infinite arcs and all the domains $\Omega_{k}$ are unbounded. For a polynomial $P(z)=a_{0} z^{n}+a_{1} z^{n-1}+\ldots+a_{n}, a_{0} \neq 0$, we can describe these infinite arcs. Suppose that $\arg a_{0}=\alpha$ and $\arg c_{m}=\beta$ and let $z_{k}(t), t>0$, be the parametric equation of one of these arcs. Then $P\left(z_{k}(t)\right)=a_{0}\left[z_{k}(t)\right]^{n}\left[1+a_{1} / z_{k}(t)+\ldots\right]$ and $\arg P\left(z_{k}(t)\right)=\beta$. In other words, $\alpha+n \arg z_{k}(t)+o(t)=\beta+2 j \pi, \lim _{t \rightarrow \infty} o(t)=0$. Hence $\lim _{t \rightarrow \infty} \arg z_{k}(t)=\frac{\beta-\alpha}{n}+\frac{2 j \pi}{n}$. Thus, the arcs $z_{k}(t)$ tend asymptotically to the rays of slope $\frac{\beta-\alpha}{n}+\frac{2 j \pi}{n}, j=0,1, \ldots, n-1$. This leads to the following theorem.

Theorem 3 Every polynomial $P$ of degree $n$ defines a partition of $\widehat{\mathbb{C}}$ into $n$ unbounded regions such that the interior of every region is mapped conformally by $P$ on $\widehat{\mathbb{C}} \backslash L$, where $L$ is a cut. The mapping can be extended analytically to L, except for a finite number of points, such that $(\widehat{\mathbb{C}}, P)$ is a branched Riemann covering of $\widehat{\mathbb{C}}$ having those points as branch points. The fundamental domains of $(\widehat{\mathbb{C}}, P)$ are bounded by arcs which tend asymptotically to $n$ rays, every two consecutive rays forming an angle of $2 \pi / n$. 
Example 6: $P(z)=\frac{z^{7}}{7}-z, P^{\prime}(z)=z^{6}-1$. The branch points are the sixth roots of unity.

We consider the polygonal line $L$ passing through each image of the zeroes of $P^{\prime}$

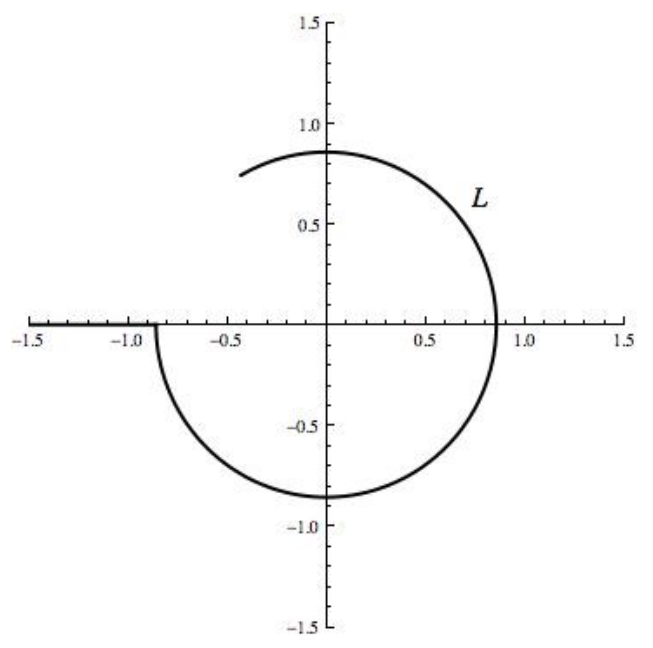

whose pre-image under $P$ is:

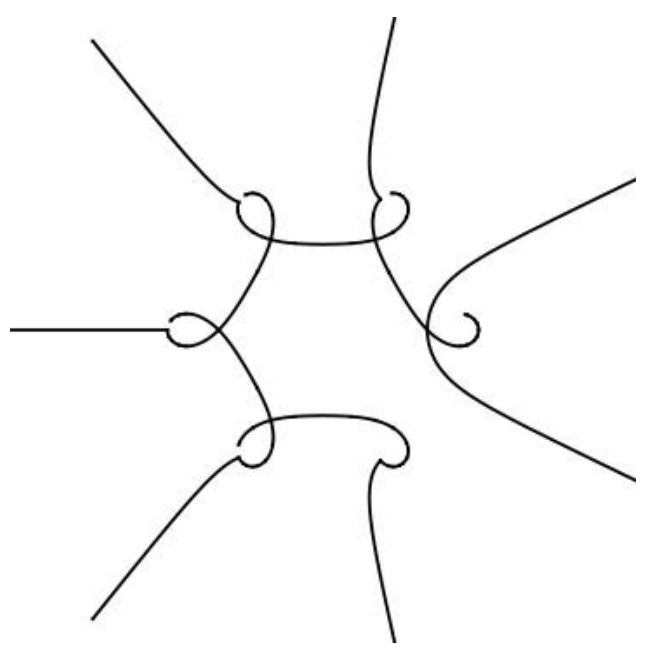


The pre-images of the annuli
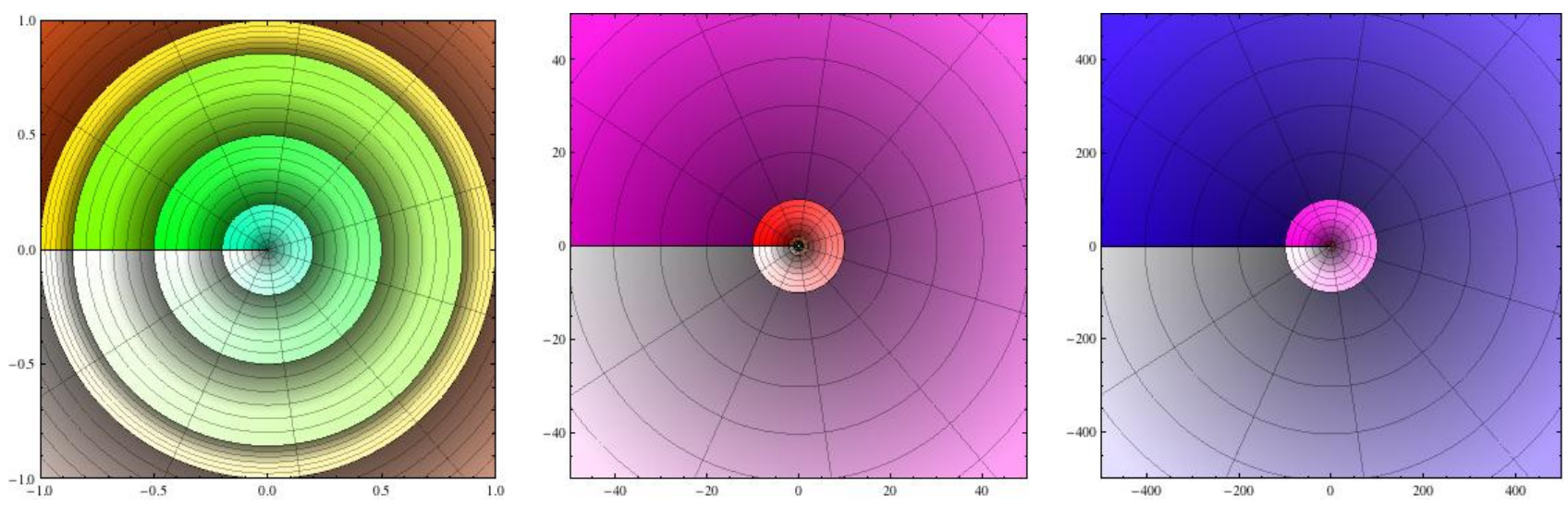

under $P$ are shown below.
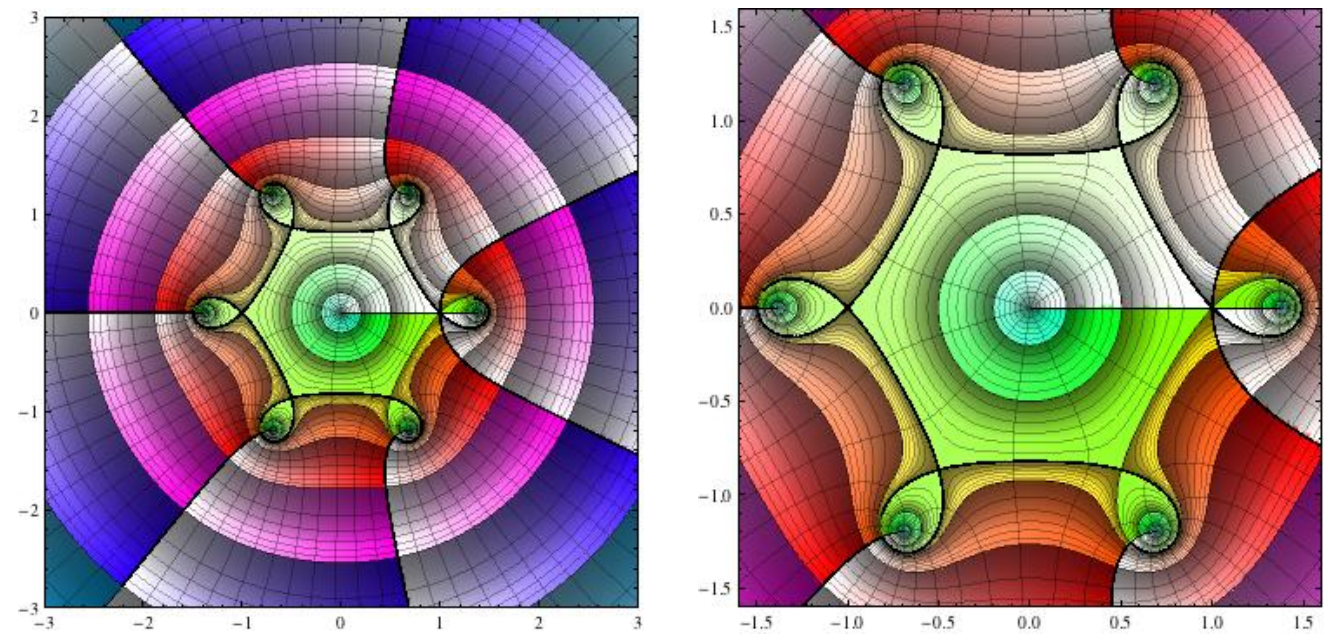

\section{References}

[A] Ahlfors, L.V., Complex Analysis, McGraw-Hill, 1979

[A-S] Ahlfors, L.V., Sario, L, Riemann Surfaces, Princeton University Press, 1960

[B-G] Ballantine, C. and Ghisa, D., Color Visualization of Blaschke Self-Mappings of the Real Projective Plane, Revue Roumaine Math. Pure Appl, 2009

[Bar-G] Barza, I, Ghisa, D., Blaschke Product Generated Covering Surfaces, Mathematica Bohemica, 2009

[N] Nehari, Z., Conformal Mappings, International Series in Pure and Applied Mathematics, 1952 